\title{
The logistics of voucher management: the underreported component in family planning voucher discussions
}

REVIEW

This article was published in the following Dove Press journal: Journal of Multidisciplinary Healthcare

\author{
Moazzam Ali' \\ Madeline Farron ${ }^{2}$ \\ Syed Khurram Azmat ${ }^{3,4}$ \\ Waqas Hameed ${ }^{5}$ \\ 'Department of Reproductive \\ Health and Research, World Health \\ Organization, Geneva, Switzerland; \\ ${ }^{2}$ School of Public Health, University \\ of Michigan, Ann Arbor, MI, USA; \\ ${ }^{3}$ Department of Uro-Gynecology, \\ University of Ghent, Ghent, \\ Belgium; ${ }^{4}$ Department of Health \\ Information Systems, Hospital for \\ Sick Children, Toronto, ON, Canada; \\ ${ }^{5}$ Research, Monitoring and Evaluation \\ Department, Technical Services, \\ Marie Stopes Society, Karachi, Sindh, \\ Pakistan
}

Background: The purpose of health care vouchers or coupons is to receive a health service in exchange which is fully or partially subsidized, such as any treatment offered for communicable disease; for immunization; antenatal care-/postnatal care-related maternal health services; a family planning (FP) service; or to get a health commodity like a medicine. Vouchers are targeted for a group of people who can benefit the most such as on the basis of poverty ranking, marginalized or living in rural areas. According to the World Health Organization, voucher schemes in the area of sexual and reproductive health are considered of high value if they are implemented to address the issues of contraceptive commodity or service unavailability or to address the barriers to access such services through contracting out health services, for example, through social franchising (SF). FP vouchers can substantially expand contraceptive access and choice and empower the underserved populations. Literature cites voucher's effectiveness in better targeting, increasing use, and improving program outcomes in FP programs; however, there is little research or explanation of how voucher management is done in practice.

Discussion: The paper attempts to describe various components of voucher management system and its functioning using example of a voucher program in Pakistan. There are challenges such as high upfront cost, targeting the appropriate clients, validation of vouchers, and quality assurance, but these can be managed with better preparation at the planning and design stage. Strong monitoring and evaluation are integral to successful implementation of the voucher program. Also, voucher interventions that are targeted and adopt a pro-poor strategy have been found to improve access to care within poor and marginalized populations. Such programs have the capacity to bridge health inequities in developing nations. Targeted voucher schemes such as those which are designed as pro-poor or pro-rural are known to reduce barriers to access for those living with poverty or for the ones considered as marginalized population. Hence, such interventions have the capacity to fulfill the gaps in health inequities, especially, in low- and/ or middle-income countries.

Conclusion: Voucher programs should report the voucher logistics and management to build a larger evidence base of best practices. All voucher schemes must be designed, implemented, and evaluated on the basis of set objectives through addressing the local context. But any voucher implementing organization also conducting the in-house voucher management simultaneously may be considered as a weakness in program design, in turn providing rationale for either failure or success of that particular voucher intervention. Therefore, separating implementation and management of a voucher initiative can lead to enhanced transparency, improved accountability, allow for independent validation of services, and facilitate compliance for payments.

Keywords: voucher management, contraception, validation, Pakistan
Correspondence: Moazzam Ali

Department of Reproductive Health and Research, World Health Organization, Appia 20, Geneva 27, $\mathrm{CH}-1211$, Switzerland

Tel +4I 79477043 I

Email alimoa@who.int 


\section{Background}

As the sun set on the Millennium Development Goals, especially Goal 5, universal access to reproductive health, has yet to be realized. Inequitable access between countries ${ }^{1}$ and within countries continues to be a problem. ${ }^{2,3}$ However, efforts have been revitalized with Sustainable Development Goal 3, "By 2030, ensure universal access to sexual and reproductive health-care services, including for family planning (FP), information and education, and the integration of reproductive health into national strategies and programmes." ${ }^{\text {"Vouch- }}$ ers have emerged as a strategy (which includes demand- and supply-side financing) to combat inequities in access to health services and have been used in sexual and reproductive health interventions. ${ }^{5,6}$ The basic premise of a voucher is that it acts as a token that can be exchanged for goods and services, in the context of health vouchers; they are exchanged for health goods or services such as contraception or sexually transmitted infection testing. ${ }^{7}$ One particular benefit of vouchers compared to other subsidies (such as general subsidies or subsidizing the price for a geographical location) is that they are better able to target specific groups with high sensitivity (reaching a higher percentage of the people who the government or actor undertaking the program wants to subsidize) and high specificity (excluding people who are not in the target group). ${ }^{7}$ It is because of these features that vouchers have been used to reach particularly underserved women in sexual and reproductive health interventions because of the ability to target specific groups, exclude those who do not qualify, and provide access for underutilized health services. Besides addressing the financial barrier, vouchers are considered as a tool to stimulate demand for health services, ${ }^{8,9}$ improve quality of services at the health facility, and provide targeted subsidies for population in need such as those who are pregnant or postpartum or for postabortion cases. ${ }^{9}$

While there are published data on health vouchers and their effectiveness in programs outcomes, ${ }^{5,6}$ there is little research or explanation of how voucher management is done in practice. Some of the data from other voucher programs suggested the use of neutral (outsourced) as compared to the in-house voucher management agency. ${ }^{10,11}$ For example, in the Kenya program, the VMA was outsourced (third party) to perform service provider quality monitoring. Unfortunately, there was actually little continuous, postaccreditation quality monitoring done, and the agency responsible did not provided any postaccreditation training or other support to improve provider quality. ${ }^{10}$ In contrast, the Uganda VMA was done in-house by the implementing organization itself, which covers all aspects of the management and quality monitoring, but questions are raised on the credibility of self-monitoring reports. ${ }^{10}$ However, no evaluation research is available to document the impact of the Kenyan as well as Ugandan voucher programs discussing the effects of outsourced vs in-house VMA. But the programmatic lessons learned document by the World Bank suggest for considering a neutral agency for voucher management, an agency which does not have links to potential service providers or with the community. ${ }^{12}$ Having a third party managing the vouchers ensures strong antifraud protection measures built into the design for voucher distribution and claims made (service provider verifications and beneficiary's home-based validation). ${ }^{11}$ Despite the slight variation in voucher programs (specific organization of the scheme, local context, and organization undertaking voucher management), this paper seeks to explain the logistics of voucher management in an intervention implemented by Marie Stopes Society (MSS) in Pakistan called "Meeting the birth spacing needs of the underserved in Punjab province in Pakistan." Using demandside financing (DSF) and social franchising (SF) approaches, the project expanded the network of Suraj Social Franchise (Suraj-SF) model (comprising private health care providers) in underserved areas of Punjab and implemented a voucher program to address the financial barriers restricting uptake of FP services. The vouchers aim to reach the financially vulnerable and underserved married women of reproductive age with unmet need for contraception, and thus address financial barriers restricting FP uptake. Female field health educators (FHEs) conduct door-to-door household visits in their respective communities, and during these visits distribute voucher to extremely poor married women of reproductive age, primarily using a local poverty assessment/ ranking tool. The clients redeem vouchers at the certified Suraj-SF clinics/centers.

The objective of this paper is to explain the voucher management of an actual voucher program in order to further the knowledge bases on the process and logistics of voucher management for FP programs. Hopefully, this knowledge could inform other sexual and reproductive health and maternal health voucher programs as well.

\section{FP voucher program management}

Total fertility rate in Pakistan is high at 3.8, and all currently married women of reproductive age are reported to experience at least one unwanted pregnancy in life. ${ }^{13}$ Presently, modern contraceptive is used by $26 \%$ of women in Pakistan, ${ }^{13}$ and this rate is even lower among poorer segments of the population. It is for this reason that this voucher program for uptake of modern contraceptives targeted lowincome women. 
The program was designed as a quasi-experimental study with pre- and post phases implemented across an intervention district in Chakwal and a control district in Bhakkar in Punjab province, Pakistan, from August 2012 to January 2015. The content of this paper is derived from program data and reporting documents from this larger study on the effectiveness of DSF, SF, and single-use vouchers in Chakwal. For the purpose of a comprehensive evaluation (which was conducted separately), a multistage sampling strategy was used to recruit currently married women aged 15-49 years as study participants both from intervention and control area. ${ }^{14}$ This paper will describe the logistics of rolling out a voucher program and how the logistics of voucher management worked in order to better understand the process.

\section{Setting up voucher management system}

Provision of FP services through the public sector has failed to meet demand for contraceptives, and cost has served as a barrier when pursing contraceptives in the private sector in Pakistan. ${ }^{15}$ A significant portion of women go to the private sector for modern contraceptives, ${ }^{13}$ where financial resources may serve as a barrier to acquiring FP services. Subsequently, MSS Pakistan took a DSF approach to their vouchers, meaning that purchasing power has been transferred to a specified group (in this case low-income women in selected areas), thereby increasing economic capacity to access health services (in this case FP services). MSS has also taken an SF approach, meaning that independent private health providers work in a network and use commercial franchising methods in order to accomplish social goals. ${ }^{15}$ MSS' SF model is called Suraj (which is "Sun" in English) and consists of, as of October 2017, more than 500 private provider partners, an output-based aid and voucher scheme, and more than 500 field worker mobilization groups in 38 districts in the country. This model has been very successful for MSS in increasing access, use, and improving quality of services. ${ }^{15-17}$ Suraj clinics are easily identifiable with a sun logo displayed. MSS used single-purpose FP vouchers that are provided at the Suraj clinics. The voucher shown in the figure indicates in Urdu language that free contraceptive services can be obtained for implants, intrauterine devices (IUDs), condoms, pills, and injectables (Figure 1).

The voucher initiative consists of the following:

1. FP services are only free

2. FP visits are prepaid (total three)

3. Both short-term and long-term contraceptive methods were provided during the visit

4. Follow-up visits for managing side effects and for removal services of FP methods, if required
Community-level providers, for example lady health visitors or equivalent and general physicians (with a basic medical degree), were trained to provide FP services, including longacting reversible methods such as IUDs and contraceptive implants (provided by qualified doctors). FHEs, who are essentially community outreach workers, assessed women for poverty and need of FP, and also counseled women for FP. Women who expressed willingness to adopt a modern contraceptive method, if they were determined to be in the bottom two wealth quintiles on the poverty scale, were offered the vouchers (for the three visits described earlier).

After receiving a voucher, a woman could redeem services at any Suraj project provider. Because mid-level providers are not allowed to insert Femplant (two-rod subdermal implant), these women from Suraj clinics are referred to a nearby franchised general physician.

\section{Voucher design and record keeping}

Vouchers are designed centrally at the MSS Head office. Each voucher has a unique number and watermark with key brand symbols to avoid counterfeit vouchers. The number series was developed by the individual in-charge (the "in-charge") of the voucher scheme, based at the social franchise technical department at MSS.

Each FP voucher has four sections that are organized into tabs. The first tab is retained by the FHE after distribution for official record and monitoring, and the remaining tabs are used by the client to redeem free services. A typical use would be the following:

1. Tab 1: FHE takes for record keeping

2. Tab 2: Receiving the method

3. Tab 3: Follow-up

4. Tab 4: Removal (only for IUD and implant)

The vouchers can only be redeemed within 6 months of issue data. If the voucher expires, a new voucher may be issued, per client's demand. Vouchers are nontransferable and can be redeemed only by the person to whom it is issued.

FHE, at the time of issuing the voucher to a woman, fills out the following information on the vouchers: intervention area code, district name, date of issue, service(s), client's and husband's name, number of children, and complete address of the client (to identify and locate client). The FHEs also keep a diary for recording the client's data, where this information is also recorded.

The women will receive free contraceptive service with the voucher. The provider also keeps a daily diary for recording the client's data, where this information is again recorded (especially the type of service provided and the unique 
A

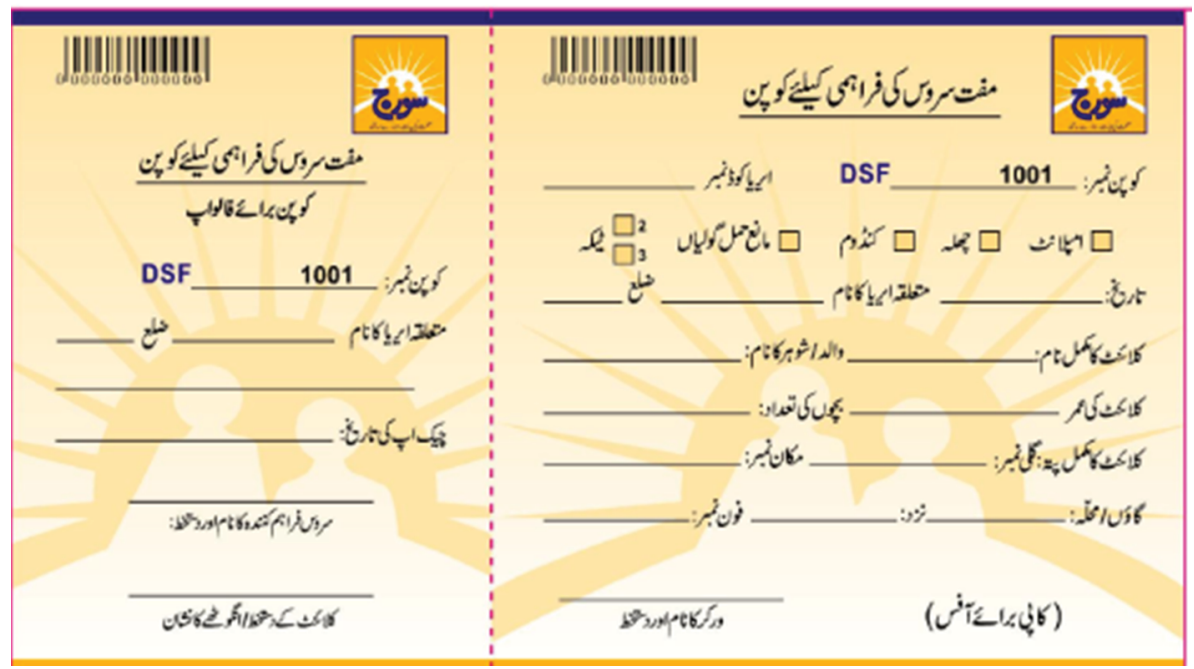

B

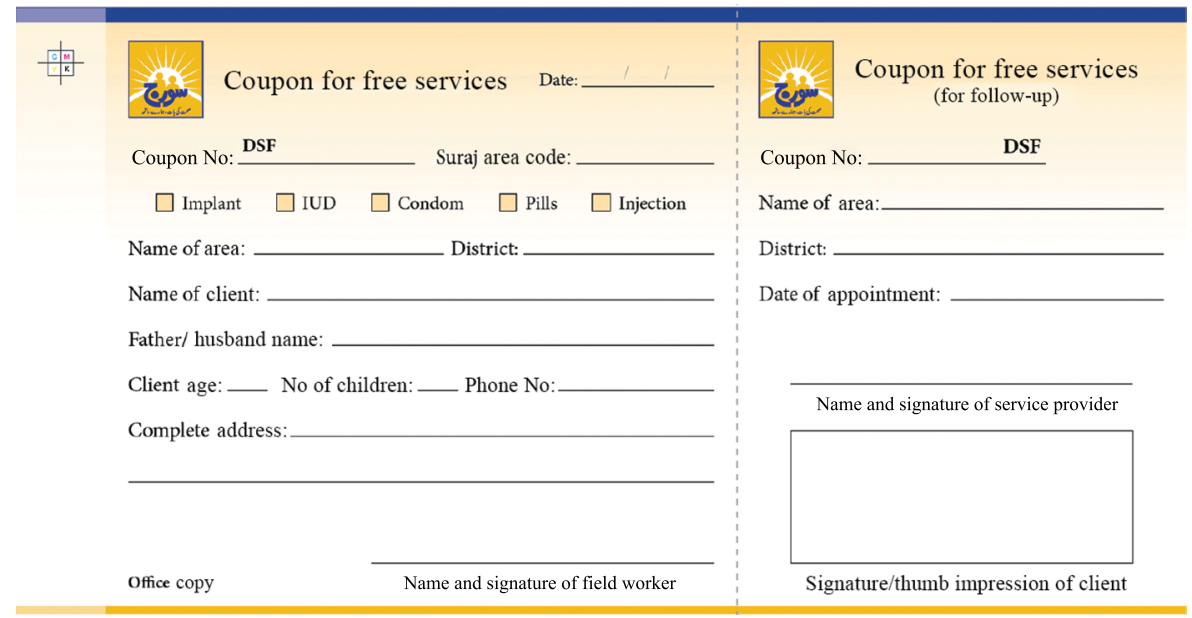

C

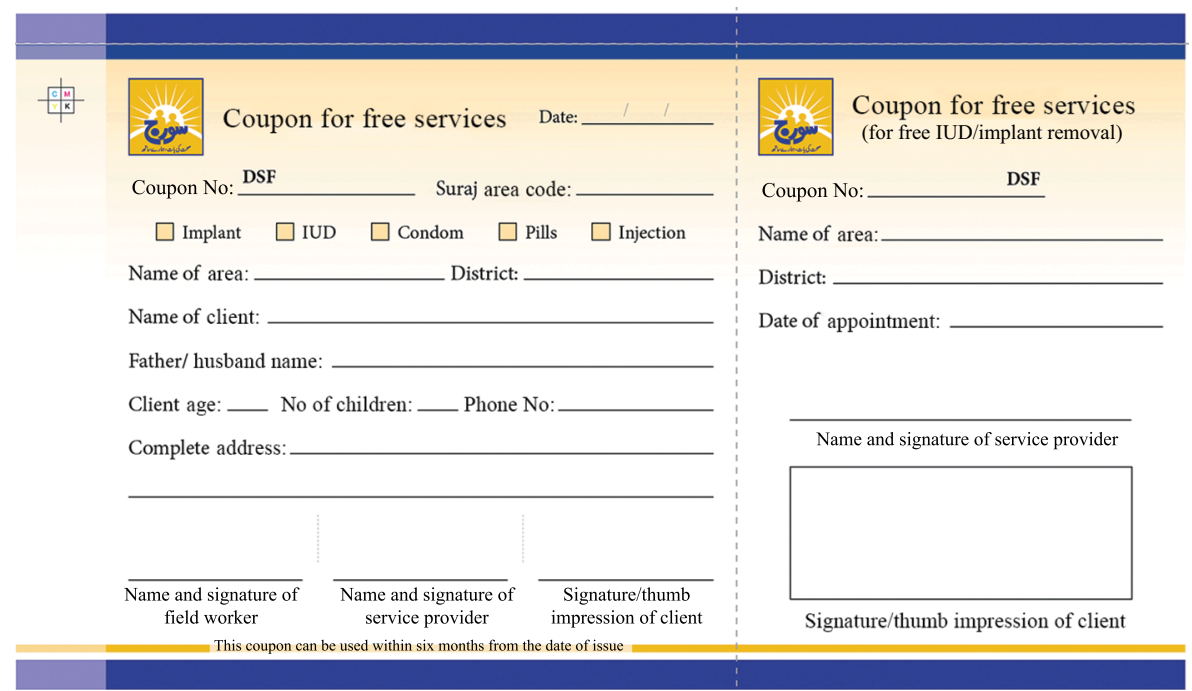

Figure I Voucher for contraception services in Urdu (A) and translated to English for follow-up (B) and for free IUD/implant removal (C). Note: The original figure was translated from Urdu to English by Waqas Hameed for inclusion in this paper.

Abbreviation: DSF, demand-side financing. 
voucher code) along with the client's name and other details. The documentation in FHE and provider daily dairies serve as a proof that the client has received the services, and the respective project office has a copy of the distributed voucher to identify that vouchers are distributed in the field during the validation process. All original voucher tabs are submitted to MSS field supervisor in each district for reimbursement.

\section{Monitoring and reporting}

There are also various spot checks for validation by the district-level operations teams. The internal audit (IA) department at MSS randomly selects redeemed vouchers for validation and reports back to the Country Director and Board of Directors at MSS. There are also penalties such as deaccreditation, for the Suraj Provider and field staff, if there is fake voucher presentation for reimbursement. The field supervisor, district in-charge, and the monitors from IA are responsible to physically verify a sample of voucher by visiting a client's house. However, the regional and the head office staff (finance and SF department) thoroughly review the documents to check for incompleteness or discrepancies.

Because the voucher is issued using the clients' eligibility assessment form, it reduces the chances of fraud, because no one else can use the voucher.

The field supervisor, under the supervision of the district "in-charge", consolidates monthly reimbursement reports and submits them to the appropriate regional office for verification and approval at the end of each month. The regional project team verifies the selected vouchers on a household basis and submits reports to the support office, after the operations team approves the necessary checks; they submit the information to the finance department by end of each month.

Part of the validation process includes the following: making sure that the vouchers are signed by the FHE, the client (if unable to write, thumb print is used), and the provider; vouchers are distributed and redeemed in the intended area; voucher is confirmed to be not fake, expired, or transferred to another client; mandatory voucher validation is attached and signed by relevant team members; and relevant authorities have signed the documents which are necessary to make voucher-based payments to Suraj providers. External validation of the vouchers is conducted yearly on a random sample. The process is multifaceted with multidirectional relationships (Figure 2).

\section{Discussion}

As described earlier, the logistics of voucher management can be complex; however, it is necessary to see examples of how voucher programs actually work in order to implement and also in order to understand the dissimilarities between different kinds of voucher or demand-side subsidy programs. There are not only some challenges that should be noted with voucher programs but also many solutions and other lessons learned that could lead to better voucher programs in the future.

First, there may be a high upfront cost involved in implementing a large voucher program like this one in Pakistan. However, analysis has shown that, overall, vouchers are effective in promoting use of modern contraception in a long run, meaning that governments should not be too deterred by initial costs. Another potential issue when running a voucher

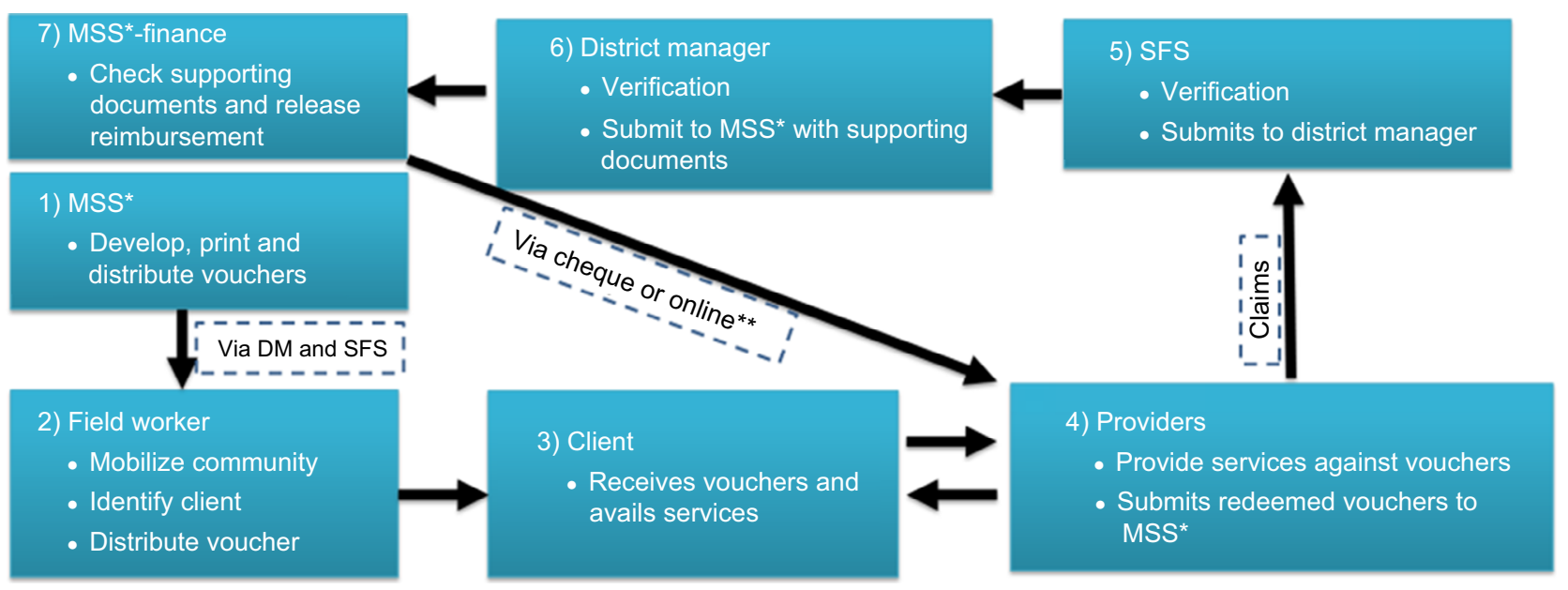

Figure 2 Voucher management system.

Notes: *MSS has an internal Voucher Management System. **No cash payment was done.

Abbreviations: DM, district manager; MSS, Marie Stopes Society; SFS, senior field supervisor. 
program is that services could be of low quality, rendering the vouchers less effective. But this can be solved with preparation. Negotiating an agreement on the quality of the services and with frequent supportive quality assurance visits, before entering the partnership, will prevent low quality services. MSS negotiates before starting the program so the quality of services remains high. Forecasting is also crucial, and it is important to have sufficient supplies of voucher books and record keeping materials before launching a program. The prep work matters in order to have a successful voucher program.

There were some challenges faced in validation including the fact that some clients were not found at home or the fact that they may have migrated, thus making it difficult to complete the validation of their voucher, and there is also difficulty in finding the exact location of the house by the external validation party. However, MSS' FHEs were often able to facilitate the location process solidifying the importance of FHEs.

The hiring of the FHE should be in coordination with the provider so that they are able to work together, but the FHE should not be related to or affiliated with the provider, thereby reducing potential conflicts of interest. A line of communication between FHEs and providers would be useful for mutual learning (provider will learn more about "the field" and FHE could learn more content knowledge from provider). In remote areas, finding qualified and trained FHEs is difficult, so training should be thorough and FHEs should be receiving continuous communication to cope with field challenges. The management of FHEs is vital to retaining FHEs. There may also be a risk to confidentiality when there are checks for verification by non-FHE employees in the voucher system. This was managed by informing and seeking consent from the client at the time of the voucher provision that organizational staff may visit them to assess satisfaction, overall experience, and to see if they were provided high quality care.

General physicians sometimes have less interest in providing FP due to fewer incentives compared to other clinical health services. This was addressed in this project with continuous behavior change communication and further close coordination and engagement with providers to maintain motivation for FP. A better package of financial compensation is required for such providers to motivate them to practice FP services. There may also be provider bias in provision of services on the voucher, as they may prefer to provide services that are more expensive. The strategy MSS took was an extensive and continuous training of health workers on FP counseling, so clients are sent for services after mobilization and are screened properly and carefully. MSS leaves much of the counseling and the voucher tender with FHE to mitigate this. However, this is a more difficult barrier to overcome. There was also some difficulty in finding time for weeklong trainings for providers, but the importance was emphasized, and many agreed to go to the full training.

Furthermore, voucher eligibility criteria could be further strengthened by targeting underserved women through a more locally (provincial or districts based) contextualized poverty assessment tool. In addition, FHE could enter live data into software, or a second layer of endorsement could be undertaken by a designated community member to ensure transparency. Another important aspect which must be considered while designing future voucher schemes is the delays caused by using a paper-based system for voucher management, which is time intensive. Organizations should opt for technological solutions and making the process electronic.

Maintaining public sector engagement at district and provincial level is key to having all actors on board. This was addressed with regular meetings with the Population Welfare Department in Punjab, Pakistan. The public sector should also be involved from the beginning, including in the selection of districts and sites, because the close coordination ensures a more successful project when each actor is involved.

Despite setbacks, this voucher program was successful as documented in several evaluations. Modern contraceptive uptake increased by $32 \%$ in the intervention group, and satisfaction with service through the vouchers was high, with a $90 \%$ satisfaction rate, ${ }^{18}$ and there was lower contraceptive discontinuation observed compared with national trend. ${ }^{19}$ Another study has shown that method discontinuation rates were not different between paid clients and voucher clients, suggesting that vouchers were correctly distributed to underserved women or else they would have discontinued for the sake of the money. ${ }^{20}$ Moreover, another study found that continuation rates were actually higher among voucher clients compared with paid clients, displaying the efficacy of vouchers. ${ }^{21}$ Moving forward, more voucher programs should report the voucher logistics and management of the voucher programs to build a larger evidence base of best practices.

\section{Conclusion}

In order to meet FP2020 commitment, voucher programs can be considered as a way to boost the use of contraception. Although FP services are provided for free at government health facilities, the model may be adopted to generate demand in the communities. This can be done through the 
national lady health worker program - the outreach workers responsible for conducting door-to-door visits for awareness rising. Certain components such as voucher management system could be outsourced to a nongovernmental organization. "Vouchers" can be used as a gateway to engage with unregulated private sector health care providers for the provision of quality services. For better accountability, sustainability, and future scale-up, it is important to consider a neutral voucher management agency for the management and monitoring of any future voucher scheme.

\section{Availability of data and material}

The datasets used and/or analyzed during the current study are available from the corresponding author on reasonable request.

\section{Author contributions}

All authors contributed toward data analysis, drafting and critically revising the paper, gave final approval of the version to be published, and agree to be accountable for all aspects of the work.

\section{Acknowledgments}

The authors are highly indebted to all the enumerators and field workers for their time and enriching this study by sharing their experiences. This work was funded by The David and Lucile Packard Foundation, USA. The funding sources did not play a role in the study design, analysis and interpretation of data, in the writing of the report, or in the decision to submit the article for publication. The conclusions and opinions expressed here are those of the authors and not necessarily those of the funder. This report comprises the shared views of a group of international experts and does not necessarily represent the decisions or the stated policy of the World Health Organization.

\section{Disclosure}

The authors report no conflicts of interest in this work.

\section{References}

1. Guttmacher Institute. Fact Sheet: Adding it Up: Investing in Contraception and Maternal and Newborn Health. New York: Guttmacher Institute; 2017.

2. Barros AJ, Ronsmans C, Axelson H, et al. Equity in maternal, newborn, and child health interventions in Countdown to 2015: a retrospective review of survey data from 54 countries. Lancet. 2012;379(9822):1225-1233.

3. Ross J. Improved Reproductive Health Equity Between the Poor and the Rich: An Analysis of Trends in 46 Low- and Middle-Income Countries. Glob Health Sci Pract. 2015;3(3):419-445.
4. United Nations Development Programme. Goal 3 Targets; 2015. New York, NY: United Nations Development Programme. Available from: http:// www.undp.org/content/undp/en/home/sustainable-development-goals/ goal-3-good-health-and-well-being/targets/. Accessed October 2, 2018.

5. Bellows B, Bulaya C, Inambwae S, Lissner CL, Ali M, Bajracharya A. Family Planning Vouchers in Low and Middle Income Countries: A Systematic Review. Stud Fam Plann. 2016;47(4):357-370.

6. Bellows NM, Bellows BW, Warren C. Systematic Review: the use of vouchers for reproductive health services in developing countries: systematic review. Trop Med Int Health. 2011;16(1):84-96.

7. World Bank. A Guide to Competitive Vouchers in Health. Washington, DC: The World Bank; 2004:128.

8. Azmat SK, Mustafa G, Hameed W, Asghar J, Ahmed A, Shaikh BT Social franchising and vouchers to promote long-term methods of family planning in rural Pakistan: a qualitative stocktaking with stakeholders. J Pak Med Assoc. 2013;63(4 Suppl 3):S46-S53.

9. Menotti EP, Farrell M. Vouchers: A Hot Ticket for Reaching the Poor and Other Special Groups With Voluntary Family Planning Services. Glob Health Sci Pract. 2016;4(3):384-393.

10. USAID. Insights from Innovations: Lessons from Designing and Implementing Family Planning/Reproductive Health Voucher Programs in Kenya and Uganda [Internet]. Bethesda, MD: Abt Associates; 2009. Available from: https://www.shopsplusproject.org/sites/default/files/ resources/5361_file_FINAL_FP_Voucher_Innovations.pdf. Accessed September 21, 2018.

11. USAID. Vouchers for Health: A Focus on Reproductive Health and Family Planning Services [Internet]. Bethesda, MD: Abt Associates; 2006. Available from: https://pdf.usaid.gov/pdf_docs/PNADI574.pdf. Accessed September 21, 2018.

12. World Bank. A Guide to Competitive Vouchers in Health. Washington, DC: World Bank. 2005:112.

13. National Institute of Population Studies (NIPS), ICF International. Pakistan Demographic and Health Survey 2012-2013. Islamabad, Pakistan: NIPS/Pakistan and ICF International; 2013.

14. Azmat SK, Ali M, Ishaque M, et al. Assessing predictors of contraceptive use and demand for family planning services in underserved areas of Punjab province in Pakistan: results of a cross-sectional baseline survey. Reprod Health. 2015;12(25):25.

15. Azmat SK, Shaikh BT, Hameed W, et al. Impact of social franchising on contraceptive use when complemented by vouchers: a quasi-experimental study in rural Pakistan. PLoS One. 2013;8(9):e74260.

16. Azmat SK, Hameed W, Hamza HB, et al. Engaging with communitybased public and private mid-level providers for promoting the use of modern contraceptive methods in rural Pakistan: results from two innovative birth spacing interventions. Reprod Health. 2016:13-25.

17. Gul X, Siddiqui J, Nasar A, Shaikh F, Gardezi L, Balal A. Social Franchising for Improving the Clinical Quality of Family Planning Services and Increasing Client Volumes at Privately Owned Clinics: Evidence from the Suraj Social Franchise Network, Pakistan, 2013-2014. Oakland, CA: Metrics for Management; 2016.

18. Ali M, Azmat S, Hamza H, editors. Assessment of the effectiveness of a demand side financing voucher schemes in meeting birth spacing needs of the underserved in Punjab, Pakistan. In: International Conference on Family Planning; January 25-28, 2016; Nusa Dua, Bali.

19. Hameed W, Azmat SK, Ali M, et al. Comparing Effectiveness of Active and Passive Client Follow-Up Approaches in Sustaining the Continued Use of Long Acting Reversible Contraceptives (LARC) in Rural Punjab: A Multicentre, Non-Inferiority Trial. PLoS One. 2016;11(9):e0160683.

20. Azmat SK, Shaikh BT, Hameed W, et al. Rates of IUCD discontinuation and its associated factors among the clients of a social franchising network in Pakistan. BMC Womens Health. 2012;12(1):12-18.

21. Hameed W, Azmat SK, Ishaque M, et al. Continuation rates and reasons for discontinuation of intra-uterine device in three provinces of Pakistan: results of a 24-month prospective client follow-up. Health Res Policy Syst. 2015;13 (Suppl 1):53. 
The Journal of Multidisciplinary Healthcare is an international, peerreviewed open-access journal that aims to represent and publish research in healthcare areas delivered by practitioners of different disciplines. This includes studies and reviews conducted by multidisciplinary teams as well as research which evaluates the results or conduct of such teams or health care processes in general. The journal covers a very wide range of areas and welcomes submissions from practitioners at all levels, from all over the world. The manuscript management system is completely online and includes a very quick and fair peer-review system. Visit http://www.dovepress.com/ testimonials.php to read real quotes from published authors. 